\title{
Infecção por Candida parapsilosis em pós-operatório de artrodese lombossacra com dispositivo de fusão intersomático TLIF em PEEK: Relato de caso
}

\section{Candida Parapsilosis Infection after Lumbosacral Arthrodesis with a TLIF Intersomatic Fusion Device in PEEK: Case Report}

\author{
Marcelo Wajchenberg ${ }^{1,20}$ Nelson Astur ${ }^{1,30}$ Michel Kanas ${ }^{1,2}$ Thiago Zinsly Sampaio Camargo ${ }^{10}$ \\ Sérgio Barsanti Wey ${ }^{1}$ Délio Eulalio Martins ${ }^{1,4}$
}

1 Hospital Israelita Albert Einstein, São Paulo, SP, Brasil

${ }^{2}$ Departamento de Ortopedia e Traumatologia, Universidade Federal de São Paulo, São Paulo, SP, Brasil

${ }^{3}$ Santa Casa de Misericórdia de São Paulo, São Paulo, SP, Brasil

${ }^{4}$ Universidade Anhembi Morumbi, São Paulo, SP, Brasil

Endereço para correspondência Marcelo Wajchenberg, MD, PhD, Av. Albert Einstein, 627/701, consultório 306, $3^{\circ}$ andar, bloco A1, Morumbi, São Paulo, SP, 5652-900, Brasil

(e-mail: marcelow@einstein.br).

Rev Bras Ortop 2021;56(3):390-393.

\section{Resumo \\ Palavras-chave \\ - infecções fúngicas \\ - espondilodiscite \\ - Candida parapsilosis}

As espondilodiscites são complicações infrequentes, porém graves em pós-operatórios de cirurgias da coluna vertebral, tendo como principal agente etiológico o Staphylococcus aureus. As infecções fúngicas são raras, sendo a Candida albicans a principal representante desse grupo. Relatamos o caso clínico de um paciente do sexo masculino, 69 anos, operado com artrodese de L2 a S1 para correção de escoliose degenerativa. O paciente apresentou quadro clínico infeccioso 2 meses e meio após o procedimento, relacionado à espondilodiscite L5-S1, causada por Candida parapsilosis. O tratamento consistiu na remoção do material cirúrgico, colocação de enxerto tricortical de ilíaco pela via anterior (L5-S1) e fixação lombopélvica (de T10 à pelve) pela via posterior, além de iniciar o tratamento medicamentoso com anidulafungina e fluconazol, mantendo essa última medicação por 12 meses, com boa evolução clínica.

Spondylodiscitis is an uncommon but serious complication after spine surgeries, and its main etiologic agent is Staphylococcus aureus. Fungal infections are rare and mostly caused by Candida albicans. We report the clinical case of a 69-year-old male patient who underwent a L2-S1 arthrodesis for degenerative scoliosis correction. He presented an infection 2.5 months after the procedure, a spondylodiscitis at L5-S1 levels, caused by Candida parapsilosis. The treatment consisted of surgical material removal, tricortical iliac graft placement in an anterior approach (L5-S1), lumbopelvic fixation (from T10 to the pelvis) in a posterior approach, and drug treatment with anidulafungin and fluconazole. This last medication was administered for 12 months, with good clinical outcomes.

Trabalho desenvolvido no Hospital Israelita Albert Einstein, São Paulo, SP, Brasil.

recebido

27 de Maio de 2020

aceito

16 de Setembro de 2020

published online

Março 30, 2021
DOI https://doi.org/ 10.1055/s-0040-1721845. ISSN $0102-3616$.
(C) 2021. Sociedade Brasileira de Ortopedia e Traumatologia. All rights reserved.

This is an open access article published by Thieme under the terms of the Creative Commons Attribution-NonDerivative-NonCommercial-License, permitting copying and reproduction so long as the original work is given appropriate credit. Contents may not be used for commercial purposes, or adapted, remixed, transformed or built upon. (https://creativecommons.org/ licenses/by-nc-nd/4.0/)

Thieme Revinter Publicações Ltda., Rua do Matoso 170, Rio de Janeiro, RJ, CEP 20270-135, Brazil 


\section{Introdução}

As infecções em pós-operatório de cirurgias da coluna vertebral são infrequentes, mas potencialmente graves, principalmente na presença de implantes, que podem perpetuar 0 processo infeccioso, mantendo quadro de espondilodiscite, que pode cronificar. ${ }^{1}$ o principal agente etiológico é o Staphylococcus aureus, mas outras bactérias como Streptococcus e bacilos gram negativos podem causar a infecção; também não se pode descartar outros germes, como o bacilo da tuberculose e, mais raramente, fungos. ${ }^{2}$ Dentre os fungos, a Candida albicans é o mais frequente, acometendo principalmente pacientes imunodeprimidos e alcoolistas. ${ }^{2}$

Apresentamos um paciente hígido de 69 anos que desenvolveu infecção em pós-operatório de artrodese de coluna lombossacra, com isolamento de Candida parapsilosis no nível L5-S1, local onde foi colocado dispositivo para fusão intersomática com estrutura em poli (éter-éter-cetona) (PEEK, na sigla em inglês).

\section{Relato do caso}

Paciente masculino, 69 anos, hígido, foi tratado de lombociatalgia esquerda clinicamente sem sucesso por mais de 6 meses, sendo operado em outubro de 2018, com descompressão neural L4-L5 e L5-S1 e retirada de hérnia discal. O paciente teve melhora parcial, mas apresentou retorno dos sintomas de forma progressiva, sem sinais de infecção.

Devido à persistência dos sintomas e da doença degenerativa da coluna vertebral e à necessidade de ampliar a descompressão neural, optou-se por ampla laminectomia L4-L5 e L5-S1, com correção da deformidade escoliótica, colocação de dispositivos intersomáticos em PEEK, enxertia óssea nos níveis L3-L4, L4-L5 e L5-S1 e fixação com parafusos pediculares bilateralmente entre L2 e S1 em janeiro de 2019.

Dois meses e meio após a cirurgia, o paciente relatou aumento progressivo de dor lombar, irradiação principalmente para o membro inferior direito (MID), associados a episódios febris. $\mathrm{O}$ paciente foi internado e foi submetido a ressonância magnética (RM), (-Figura 1) que mostrou sinais de espondilodiscite em L5-S1, além de presença de coleção (abscesso) dentro do canal vertebral, comprimindo o saco dural.

Baseado no quadro clínico e no referido exame, foi realizada cirurgia para descompressão neural, mantendo os implantes, drenagem de coleção infecciosa, e material foi enviado para análise microbiológica, a qual evidenciou crescimento de Candida parapsilosis.

Com esse diagnóstico, iniciou-se o tratamento específico com drogas antifúngicas endovenosas, anidulafungina e fluconazol, havendo boa resposta clínica. Após 20 dias, o paciente voltou a sentir dores e piora do estado clínico geral. Tomografia computadorizada (TC) (-Figura 2) e RM (-Figura 3) de controle mostraram aumento de coleção no espaço discal L5-S1 e sinais de soltura dos parafusos de S1, principalmente à direita.

Dessa forma, 1 mês e meio após a limpeza cirúrgica que evidenciou o germe, realizou-se nova cirurgia, com abordagem inicial pela via posterior, para retirada dos parafusos de L5-S1, extensão da fixação à pelve com parafusos de ilíaco e retirada de enxerto da crista ilíaca para utilização na abordagem pela via anterior.

Em seguida, o paciente foi posicionado em decúbito dorsal e foi realizada abordagem retroperitoneal para retirada do dispositivo intersomático (TLIF) L5-S1, que se apresentava solto, sem sinais de consolidação. Foi realizada extensiva limpeza

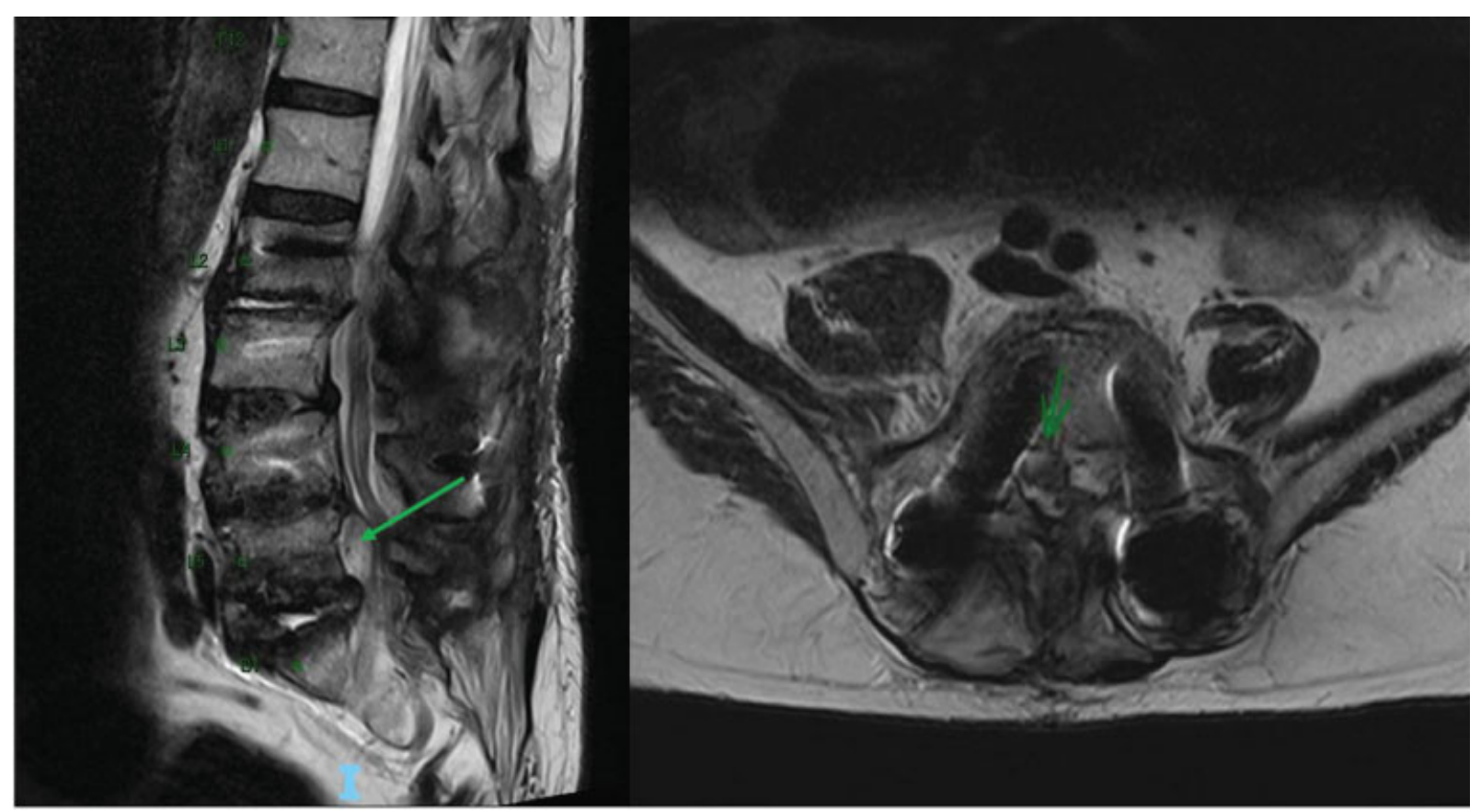

Fig. 1 Imagens de ressonância magnética ponderada em T2 (sagital à esquerda e axial à direita) mostrando a presença de abscesso posterior ao corpo vertebral de L5. 


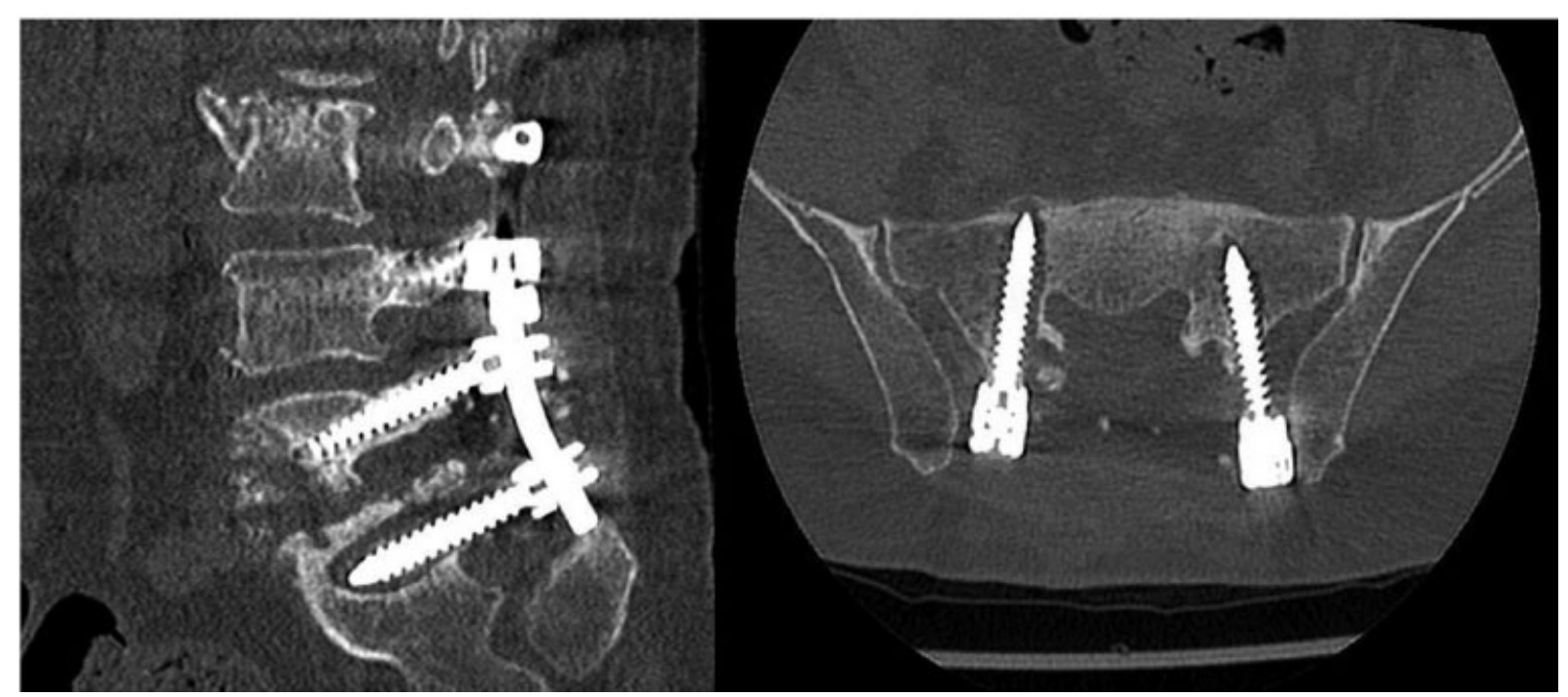

Fig. 2 Tomografia computadorizada, corte sagital à esquerda e axial à direita, mostrando sinais de soltura dos parafusos pediculares em S1, principalmente a D.

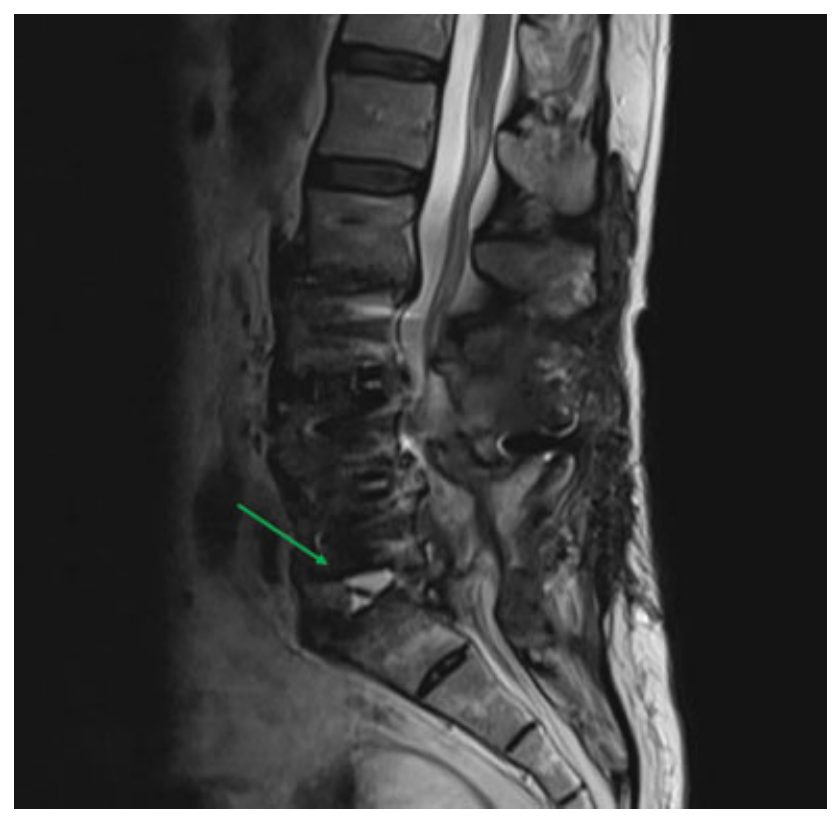

Fig. 3 Imagem sagital de ressonância magnética ponderada em T2 mostrando hipersinal no espaço discal L5-S1 referente a coleção líquida local.

cirúrgica, desbridamento, coleta de material para cultura e colocação do enxerto tricortical de ilíaco, sob pressão.

O material coletado durante a cirurgia confirmou a presença de $C$. parapsilosis. Após a cirurgia, o paciente foi tratado clinicamente com fluconazol durante 12 meses, sem intercorrências e melhora gradual, realizando exames de controle (Rx e RM de coluna lombossacra), evidenciando sinais de consolidação da artrodese lombossacra (-Figura 4).

\section{Discussão}

As infecções fúngicas na coluna vertebral ocorrem principalmente em indivíduos com algum grau de imunodeficiência, que pode ser causado pelo consumo excessivo de álcool ou drogas, desnutrição ou doenças consumptivas. ${ }^{1-4}$ Segundo Colombo, a maioria dos casos de infecção sistêmica por Candida spp ocorre por via endógena, com a translocação do patógeno através do trato gastrointestinal, local onde há rica colonização por Candida spp em até 70\% da população normal. ${ }^{4}$ Isso pode ocorrer no pós-operatório de cirurgias de grande porte, devido ao uso de antibióticos e íleo paralítico.

Outra forma de contaminação pode ser por via exógena, por meio do contato das mãos de profissionais de saúde em pacientes portadores de cateteres vasculares na posição central, implante de próteses contaminadas, bem como pela administração parenteral de soluções contaminadas. ${ }^{4}$

No caso apresentado, as queixas do paciente foram: febre, aumento de dor na região lombar e diminuição de força nos membros inferiores; relacionadas à formação de coleção (abscesso) no canal vertebral. Tais sinais foram relatados por Cho et al., ${ }^{1}$ salientando que até $20 \%$ dos pacientes poderiam ter piora do quadro neurológico.

Em relação ao tratamento específico, existem poucos relatos que envolvem processo infeccioso associado à presença de algum tipo de implante. Segundo Colombo et al., ${ }^{4}$ a C. parapsilosis prolifera em soluções de glicose e tem grande capacidade de produzir biofilme, justificando a falta de resposta clínica adequada, somente com uso de medicações específicas e limpeza cirúrgica sem retirada do implante.

Blecher et al. ${ }^{3}$ relataram um caso de paciente etilista crônico que desenvolveu infecção por $C$. parapsilosis após colocação de implante tipo eXtreme lateral interbody fusion (XLIF) (também em PEEK), por meio de acesso minimamente invasivo (anterolateral), no nível L3-L4, com artrodese prévia de L4-S1.

Richaud et al. ${ }^{5}$ observaram em série de 28 pacientes com espondilodiscite por Candida que a utilização de medicação antifúngica por período mais prolongado ( $>6$ meses) propiciou menos óbitos e complicações neurológicas e que o uso 


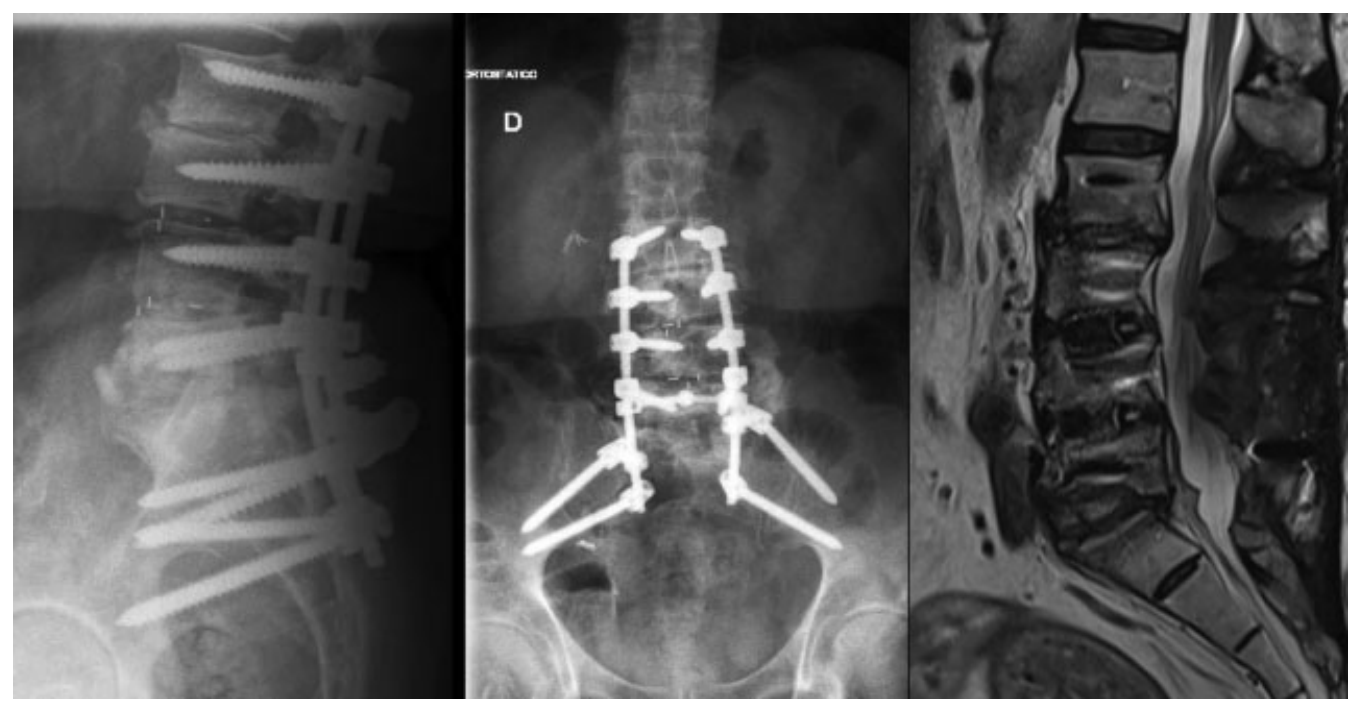

Fig. 4 Radiografias de coluna vertebral em perfil (esquerda) e de frente (centro), mostrando sinais de artrodese (fusão entre L2 e S1) e imagem de ressonância magnética (direita) ponderada em T2, corte sagital, mostrando sinais de resolução do processo infeccioso.

inicial da combinação de medicações terapêuticas proporcionou melhor evolução, com menos sequelas.

A espondilodiscite causada por C. parapsilosis é uma grave complicação da cirurgia da coluna vertebral, devendo ser diagnosticada e tratada de forma precoce, com abordagem cirúrgica, para drenagem de abscesso e coleta de material para identificação do germe e com a terapêutica medicamentosa adequada, usando duas drogas específicas inicialmente e mantendo fluconazol por $>6$ meses.

\section{Suporte Financeiro}

Não houve suporte financeiro de fontes públicas, comerciais, ou sem fins lucrativos.

\section{Conflito de interesses}

Os autores declaram não haver conflito de interesses.

\section{Referências}

1 Cho K, Lee SH, Kim ES, Eoh W. Candida parapsilosis spondylodiscitis after lumbar discectomy. J Korean Neurosurg Soc 2010;47 (04):295-297

2 Kulcheski AL, Graells XS, Benato ML, Santoro PG, Sebben AL. Espondilodiscite fúngica por Candida albicans: um caso atípico e revisão da literatura. Rev Bras Ortop 2015;50(06):239-242

3 Blecher R, Yilmaz E, Moisi M, Oskouian RJ, Chapman J. Extreme Lateral Interbody Fusion Complicated by Fungal Osteomyelitis: Case Report and Quick Review of the Literature. Cureus 2018;10 (05):e2719

4 Colombo AL, Guimarães T. [Epidemiology of hematogenous infections due to Candida spp]. Rev Soc Bras Med Trop 2003;36(05): 599-607

5 Richaud C, De Lastours V, Panhard X, Petrover D, Bruno F, Lefort A. Candida vertebral osteomyelitis (CVO) 28 cases from a 10-year retrospective study in France. Medicine (Baltimore) 2017;96(31): e7525 